\title{
Covid-19: Hydroxychloroquine does not benefit hospitalised patients, UK trial finds
}

\author{
Ingrid Torjesen
}

London, UK

Hydroxychloroquine does not reduce the risk of dying or improve other outcomes in hospitalised patients treated for covid-19, preliminary results from the UK RECOVERY trial have shown.

Announcing the results, Martin Landray, the study's deputy chief investigator, said, "We have concluded that there is no beneficial effect of hydroxychloroquine in patients hospitalised with covid-19. We have therefore decided to stop enrolling participants to the hydroxychloroquine arm with immediate effect."

The data show the death rate at 28 days in covid-19 patients taking hydroxychloroquine is $25.7 \%$ compared with $23.5 \%$ in patients provided with only usual hospital care (hazard ratio 1.11 (95\% confidence interval 0.98-1.26); $\mathrm{P}=0.10$ ). So far 1542 patients in the trial have been randomised to hydroxychloroquine and 3132 patients randomised to usual care alone.

There was also no evidence that hydroxychloroquine impacted on length of hospital stay or had any beneficial effects on other outcomes, or that it had any beneficial effects only in certain patient groups.

The RECOVERY trial began in March and is a dynamic trial assessing candidate treatments for covid-19 in patients in UK hospitals. ${ }^{1}$ Treatments selected for the trial are chosen because there is reason to believe that they might work, they have a known safety profile, and there is sufficient supply to enable its inclusion. More than 11000 patients have been enrolled.

Reviews of the emerging data are conducted regularly by the Data Monitoring Committee to ensure that any treatments benefiting patients are picked up quickly so they can be rolled out, and that those not having a positive impact can be dropped.

On 4 June, prompted by the retraction of a paper in the Lancet on the use of hydroxychloroquine, ${ }^{2}$ the UK Medicines and Healthcare Products Regulatory Agency asked the independent Data Monitoring Committee to conduct an additional review. It then asked the chief investigators to look at the unblinded data in the hydroxychloroquine arm.

A huge upsurge in hospitalised cases around a month ago meant that the amount of data on hydroxychloroquine had increased very recently and "the picture has changed," said Landray, professor of medicine and epidemiology at the Nuffield Department of Population Health at the University of Oxford.

"There are hundreds of thousands, potentially millions, of patients around the world being treated with hydroxychloroquine," he said. "This treatment does not reduce the risk of dying from covid-19 among hospital patients-that clearly has a significant importance for the way that patients are treated not only in the UK but around the world."

Last week a Lancet paper, $^{2}$ which suggested that use of hydroxychloroquine to treat covid-19 could increase risk of death, was retracted because of questions over the provenance of the data. ${ }^{3}$ The paper had prompted the World Health Organization to pause the hydroxychloroquine arm of its Solidarity trial which is testing possible treatments for covid-19, although it reversed this decision following the retraction. ${ }^{4}$

The RECOVERY trial's chief investigator Peter Horby, professor of emerging infectious diseases and global health in the Nuffield Department of Medicine, University of Oxford, said that he had spoken to WHO about the results. "They will be convening their committee to reconsider their decision based on the events of today," he said.

Several countries, including the US have made the drug available for emergency use in hospitalised covid-19 patients, and President Donald Trump has promoted it and revealed that he is taking it prophylactically.

The RECOVERY trial is by far the biggest trial that will report on hydroxychloroquine in covid-19, said Landray. While the data were clear that there were no meaningful benefits as a treatment for hospitalised patients, they could not be extrapolated to cover different settings or preventative use, he said, because patient populations, disease stage, and dosages would be different.

Last week a study published in the New England Journal of Medicine found hydroxychloroquine was ineffective as prophylaxis. $^{5}$

Five other treatments continue to be assessed by the RECOVERY trial: lopinavir-ritonavir, low dose dexamethasone, azithromycin, tocilizumab, and convalescent plasma (collected from donors who have recovered from covid-19). Results on these are expected in early July.

\footnotetext{
Wilkinson E. RECOVERY trial: the UK covid-19 study resetting expectations for clinical trials. BMJ 2020;369:m1626. 10.1136/bmj.m1626 32345591

2 Lancet. Expression of concern: Hydroxychloroquine or chloroquine with or without a macrolide for treatment of COVID-19: a multinational registry analysis. https://marlin-prod. literatumonline.com/pb-assets/Lancet/pdfs/S0140673620312903.pdf.

lacobucci G. Covid-19: Validity of key studies in doubt after leading journals issue expressions of concern. BMJ 2020;369:m2224. 10.1136/bmj.m2224 32499292

4 Rahhal N. WHO will resume trials of hydroxychloroquine to treat coronavirus as experts question studies that suggested the malaria drug raises risks of death. MailOnline. 3 June 2020

5 Mahase E. Covid-19: Hydroxychloroquine was ineffective as postexposure prophylaxis, study finds. BMJ 2020;369:m2242. 10.1136/bmj.m2242 32503805
} 
Published by the BMJ Publishing Group Limited. For permission to use (where not already granted under a licence) please go to http://group.bmj.com/group/rights-licensing/

permissions 